


\title{
El hombre como "el animal enfermo": sobre el significado de salud y enfermedad en la antropología crítica de Nietzsche ${ }^{1}$
}

\section{The human being as "the sick animal": on the meaning of health and illness in Nietzsche's critical anthropology}

\author{
Diana Aurenque \\ Universidad de Santiago de Chile, Chile
}

\begin{abstract}
Resumen: En las últimas décadas, y gracias a la publicación de trabajos de autores como Jacques Derrida y Giorgio Agamben, se ha reactivado y replanteado en el mundo filosófico la clásica pregunta por la relación entre lo humano y lo animal. Particularmente, la filosofía de Friedrich Nietzsche proporciona uno de los ejemplos más notables donde la relación entre existencia y animalidad ha sido esbozada en términos tanto de cercanías y lejanías fisiológico-empíricas como también teóricas, proporcionándonos un modo sumamente novedoso y del todo productivo de afrontar la relación humano/animal. En este sentido, el presente trabajo tiene por fin los siguientes objetivos: 1) En primer lugar, la contribución contextualiza y examina los alcances y las consecuencias de la definición Nietzscheana del hombre como "el animal enfermo" ("das kranke Tier"), para luego, 2) interpretar el significado tanto médico-biológico como también metafórico de la salud y enfermedad, como ámbitos agonísticos, para con ello 3) evaluar la singularidad de la forma de ser "enferma"
\end{abstract}

${ }^{1}$ El presente trabajo se enmarca en el proyecto FONDECYT DE INICIACIÓN Nro. 11150298 del cual soy investigadora Responsable, y también en parte en el proyecto FONDECYT Nro. 1120730. Para asuntos de edición del presente texto agradezco al Sr. Rodrigo Lagos. 
del hombre. Finalmente, el estudio se pregunta y evalúa 4) hasta qué punto dicho planteamiento efectivamente representa un avance para el estudio de los límites entre hombres y animales.

Palabras claves: salud y enfermedad, filosofía de la medicina, Nietzsche, "animal enfermo", antropología filosófica.

Abstract: In recent decades, and due to the publication of works from authors such as Jacques Derrida and Giorgio Agamben, the classical question about the relationship between the human and the animal has been reactivated and rethought in the philosophical world. In particular, Friedrich Nietzsche's philosophy provides one of the most interesting example in which the relationship between human existence and animality has been described, providing us with an extremely original and productive way of dealing with this relationship. The present work aims the following objectives: 1) In the first place, the contribution contextualizes and examines the scope and consequences of the Nietzschean definition of man as "the sick animal" ("das kranke Tier"), 2) to interpret both the medical-biological as well as the metaphorical meaning of health and disease as agonistic expressions, in order to 3 ) assess the uniqueness of man's "sickness". Finally, the study asks and evaluates 4) to what extent this approach effectively represents an advance for the anthropological questions.

Keywords: Health and disease, Philosophy of medicine, Nietzsche, Sick animal, Philosophical Anthropology.

\section{Introducción}

Como es conocido, Paul Ricoeur denomina a Friedrich Nietzsche al igual que a Sigmund Freud y Karl Marx uno de los "maestros de la sospecha”. De acuerdo con Ricoeur, estos pensadores plantean críticas profundas al paradigma antropológico que desde Alcmeón de Crotona en adelante -y al menos hasta las filosofías trascendentales de Immanuel Kant y Georg Wilhelm Friedrich Hegel- aboga por diferenciar al ser humano de los animales por tener acceso al logos. Sin embargo, con el enorme desarrollo de la 
ciencia natural, en especial de la fisiología del siglo XIX, y gracias al igualmente descomunal impacto de la teoría evolutiva de Charles Darwin, surge la necesidad de repensar la forma de entender y definir al hombre. De la mano a esto, se establece una fuerte tendencia materialista y anti-metafísica en la filosofía, que tiene por fruto una renaturalización, y en sentido estricto, una reanimalización del hombre.

La filosofía de Nietzsche sigue precisamente esta tendencia y no sólo rehabilita la pertenencia de lo humano a lo animal, sino que desvaloriza lo humano de manera radical (Cf. Lemm, 2012; 2014; 2016). En diversas ocasiones, como mencionaremos, Nietzsche definirá al hombre como una especificación de lo animal: como enfermo, absurdo, no establecido, etc. En particular, su definición del hombre como "el animal enfermo" (das kranke Tier) y que es mencionada en la Genealogía de la moral, no sólo resalta el lado animal del hombre, sino que al mismo tiempo, con esta definición, Nietzsche lo crítica por representar una versión deficiente de animalidad -aquí por ejemplo se observan las fuentes Nietzscheanas de la antropobiología de Arnold Gehlen y su definición del hombre como "ser deficitario" (Gehlen, 2016). En la definición del hombre como "animal enfermo", el campo de diferenciación y de cercanía entre hombre y animal ya no se relaciona a aspectos cognoscitivos y/o de su tener cabida en el logos griego, sino permaneciendo en una línea materialista y naturalista, Nietzsche lo definea partir de los conceptos de salud y enfermedad.

En vistas de lo anterior, la presente contribución tiene los siguientes propósitos: 1) En primer lugar, contextualiza y examina los alcances y las consecuencias de la definición Nietzscheana del hombre como "el animal enfermo", para luego 2) interpretar el significado tanto médico-biológico como también metafórico de la salud y enfermedad como ámbitos agonísticos, para con ello 3) evaluar la singularidad de la forma de ser "enferma" del hombre. 
Finalmente, 4) también debe reflexionar hasta qué punto dicho planteamiento efectivamente representa un avance para el estudio de los límites entre hombres y animales.

\section{El hombre como "animal enfermo"}

En diversas obras Nietzsche no cesará de insistir que el hombre no es más que un "camino" (Weg), un "puente" (Brücke) como dirá en su Zaratustra (Nietzsche, 1999c: 16), es decir, algo que debe ser superado. En concordancia con su enseñanza del superhombre o sobrehombre (Übermensch) lo humano es postulado como algo transitorio y deficiente. En estas descripciones de lo que se puede llamar la antropología crítica de Nietzsche, el filósofo hace uso recurrente de una metafórica médica. En efecto, el mismo Nietzsche se autodenomina "médico" (Arzt) y a su filosofar como un "ciencia médica" (medicinische Wissenschaft). Por ello, no es casual que algunos comentadores de la obra de Nietzsche hablen de su "metafórica médica" (medizinische Metaphorik; Brusotti, 2001), de su "filosofía médica" (philosophie médicale; Cherlonneix, 2002) o incluso de su "filosofía de la medicina" (philosophy of medicine; Long, 1990). En diversas obras, Nietzsche se refiere al hombre y a lo humano como algo "enfermo", como algo malsano y necesitado de una nueva salud. A ello se debe que en la investigación actual sobre Nietzsche existe una amplia literatura que tematiza la relación entre su pensar y la medicina (Cf. Friedrich et. al., 2016; Aurenque/Friedrich, 2013; Aurenque, 2013a; 2013b; Schipperges, 1975; 1981; 1985; Sorgner, 2009; 2010; 2011).

En concordancia con la filosofía anti-esencialista y contraria a cualquier dualismo metafísico, Nietzsche define la forma de ser del ser humano desde una estructura inevitablemente ambivalente. Por un lado, el hombre pertenece al reino de la naturaleza; por ello, Nietzsche lo llama "animal" o en otras obras incluso, "bestia". 
En tanto el humano es definido como una modificación o especificación de lo animal, pareciera ser cierta la tesis de Max Scheler acerca de que el concepto de hombre casi siempre está "subordinado" al concepto de animal (Scheler, 1997: 24). Nietzsche, como vemos, sigue a su modo la tradición para luego transformarla. En El Anticristo sostiene: "No derivamos más al hombre del "espíritu", de la "divinidad", lo hemos re-posicionado entre los animales" (Nietzsche, 1999b: 180). Por otra parte, el ser del hombre no se reduce a su esencia animal y/o natural, sino que él se constituye en tanto humano fundamentalmente y sólo gracias a la disfuncionalidad de su constitución animal. Así, el hombre se diferencia de los demás animales justamente a causa de la plasticidad de su esencia, como un ente de algún modo auto-construido. Como lo entiende Nietzsche, el humano consiste en una ambivalencia: por un lado, en un "animal", mas por otro, le pertenece exactamente a su volverse humano la tarea de negar su propio origen animal. Dicha negación -que jamás puede ser absoluta- consiste en situar la animalidad del hombre bajo un sistema normativo y, por tanto, propiamente humano (representado, por ejemplo, por la socialización, la moralidad y/o la religión). Por naturaleza, de acuerdo con Nietzsche, estamos condenados a desarrollar una segunda naturaleza que está obligada a interpretar y revalorar los fundamentos vitales en función de algo ajeno a la primera naturaleza; a saber, el mundo humano y sus diversos entramados normativos.

La definición nietzscheana del hombre como un animal "enfermo" debe ser comprendida en el contexto recién explicado. El hombre, nos dice Nietzsche, es un "animal enfermo" ya que éste corresponde a aquel animal que si bien ha "osado, innovado, desafiado, afrontado el destino más que todos los animales juntos” (Nietzsche, 1999d: 411; 2014: 177), paradójicamente y pese a ello, denota una especial forma de carencia. La ambigüedad de Nietzsche es palpable: el hombre constituye un animal en tanto 
está conectado con la naturaleza mediante necesidades biológicas elementales (instintivas o incluso psicológicas como las llama Nietzsche); sin embargo, del mismo modo, el hombre existe en una disonancia con dichos instintos. En la Genealogía de la Moral, Nietzsche evalúa esta situación de la siguiente forma: "Pues el hombre está más enfermo, es más inseguro, más alterable, más indeterminado que ningún otro animal, no hay duda de ello, -él es el animal enfermo-" (Nietzsche 1999d: 411; 2014: 232). Esta cita es muy interesante ya que permite adentrarnos en la original forma en la que piensa Nietzsche la cercanía y la diferencia entre lo humano y lo animal.

Evidentemente, mientras que la primera consideración antropológica del hombre como a) animal no es original (pues ya está contenida en el zoon logon echon o en el zoon politikon aristotélico), sí lo es su especificación con el adjetivo b) enfermo. Como animal "enfermo" Nietzsche describe al hombre como una manifestación deficiente de la animalidad. Por último, dicha manifestación "enferma”, carente o deficitaria de animalidad, no parece ser un caso más entre otros, sino que el hombre constituye 3) un caso particular, pues Nietzsche destaca el artículo definido en cuestión al hacer referencia a "el animal enfermo" ¿Qué significan estas tres características antropológicas que nos ofrece Nietzsche?

En primera instancia, la enfermedad del hombre refiere a una salud implícita del animal. En tanto el hombre es " $e l$ animal enfermo", el animal corresponde en oposición al animal sano, dada la forma en la que éste último se relaciona con el ámbito de lo puramente natural y/o biológico. El hombre como "animal enfermizo" (Nietzsche, 199d: 367; 2014: 177) refiere a una manifestación deficiente del reino natural ya que el humano no se orienta en el mundo en relación a estructuras instintivas al modo del animal. Mientras que los animales actúan siguiendo instintos, dando rienda suelta a sus impulsos o fuerzas vitales, los humanos actúan 
en virtud de diversos afectos, intereses y motivaciones que no necesariamente están en armonía ni con ecosistemas (Ruffin 2008: 146) ni con las propias fuerzas o instintos vitales. La construcción del mundo humano, considerando sus diversas capas de organización cultural, técnica, política y social, corresponden a un entorno que dista considerablemente del entorno natural del animal, y que Nietzsche considera posible solamente por la condición enferma de lo humano, de su incapacidad de vivir saludablemente en la naturaleza como los demás animales. A partir de la falta de orientación en el mundo, Nietzsche deriva que la existencia humana se ve desorientada y siempre abrumada por la necesidad de dar con un sentido que guíe su vida.

De acuerdo con Nietzsche, el hombre como "animal enfermo" no es capaz de alinear su existencia mediante mecanismos naturales con los que ha sido dotado biológicamente. El significado del adjetivo "enfermo" evoca en primera instancia un significado particular de enfermedad. La poca orientación biológica y, producto de ello, la necesidad de construir una segunda cartografía para la propia existencia, nos muestra que la enfermedad ligada al homo humanus corresponde para Nietzsche a una suerte de "enfermedad existencial' (Jaspers, 1950) que le acontece únicamente al humano precisamente producto de la falta de sentido que rodea su existencia. En la Genealogía nos dirá Nietzsche precisamente que la falta de sentido constituye el sufrimiento más desolador para el ser humano. El sufrir sin razón, sin una causa aparente o real, sin una narrativa que de algún modo explique o justifique el sufrimiento, representa según Nietzsche el peor de los sufrimientos. No se trata con esto que el dolor o el sufrir sean considerados en sí como fenómenos negativos, sino en tanto inexplicables: "El hombre, el animal más valiente y más acostumbrado a sufrir, no niega en sí el sufrimiento: lo quiere, lo busca incluso, presuponiendo que 
se le muestre un sentido del mismo, un para-esto del sufrimiento" (Nietzsche, 1999d: 411).

El hombre es "el" animal enfermo, es decir, el único animal que vive la enfermedad en este sentido de carencia vital. Por eso nos dirá Nietzsche, sin prejuicio de la animalidad propia del hombre, que éste a fin de cuenta sólo es humano, demasiado humano. Esto quiere decir, que cuando habla de la enfermedad del hombre, no utiliza estos términos refiriéndose solamente a la enfermedad o a la salud bajo una óptica empírico-naturalista, sino también en el marco de una concepción más bien holística de la salud y la enfermedad. Así, se descubre una importante cercanía y diferencia entre lo humano y lo animal considerando ambos conceptos: mientras que el animal, hasta donde es posible tener acceso epistémico, conoce la enfermedad sólo desde el sufrir empírico y positivo, es decir, desde la experiencia del dolor o desde la discapacidad producto de una disfunción biológica y/o anatómica, el hombre no sólo es capaz de enfermar en esos términos, sino que también le es propia una forma especial de enfermedad, a saber, de tipo existencial. Esta última se acercaría a lo que ocasiona un sufrimiento vital distinto del dolor positivo. La enfermedad considerada como una carencia de sentido, afecta solamente a lo humano y a su existencia, siendo el hombre nuevamente definido como una modificación carente de lo animal. Pues, comparado con el animal el hombre es un animal absurdo justamente por el absurdo existencial que significa su ser vivo, consciente, finito y no explicado desde una narrativa metafísica apologética. Quizás uno de los testimonios más claros de habla hispana, donde se relata un absurdo existencial muy similar al que plantea Nietzsche, queda representado en los primeros versos del conocido poema de Rubén Darío "Lo Fatal":

Dichoso el árbol, que es apenas sensitivo, y más la piedra dura porque ésa ya no siente, pues no hay dolor más grande que el dolor de ser vivo 
ni mayor pesadumbre que la vida consciente.

Darío expresa una convicción no sólo de Nietzsche, sino una ya compartida por su maestro Schopenhauer: a mayor grado de conciencia, mayor grado de sufrimiento. Ahora bien, la enfermedad misma del ser humano también representa, bajo otra óptica, una importante fortaleza. Pues pese a que Nietzsche denomina al hombre un animal enfermo y también a veces absurdo, en el Anticristo sostiene que, pese a ello, también constituye “¡el más interesante!” entre los animales (“das interessanteste?”; Nietzsche, 1999b: 180). Esto tiene el propósito de resaltar las posibles dificultades, pesares e incluso dolores que conlleva la existencia humana, precisamente por el hecho de ser existencia humana y no vida animal. Existencia, absurdo y enfermedad como carencia existencial son así descubiertos como sinónimos. De forma inversa, salud y orientación parecieran entonces decir cosas que atañen en particular al animal. Hasta qué punto salud y enfermedad en el sentido Nietzscheano nos permite entender más y mejor la relación entre hombres y animales constituye el próximo punto a tratar.

\section{Salud y enfermedad como campos agonísticos}

La Real Academia de la Lengua Española define el adjetivo "agonal" (del latín agonālis) de tres formas: 1) "Perteneciente o relativo a los certámenes, luchas y juegos públicos, tanto corporales como de ingenio"; 2) "Perteneciente o relativo al combate; que implica lucha"; 3) "Se dice de las fiestas que se dedicaban al dios Jano o al dios Agonio" (RAE online). Considerando estos significados, la relación entre salud y enfermedad puede ser entendida desde una perspectiva agonal; es decir, como una relación en la que ambos conceptos se encuentran en un estado de pugna. Independientemente de las múltiples maneras que existen para definir 
estos términos, la mayoría de las teorías en este respecto entienden la relación entre salud y enfermedad como y a partir de una oposición.

Pasando revista a la literatura en torno al tema, llama la atención que la salud ha sido definida en relación a conceptos a veces incompatibles entre sí. Por ejemplo, si bien ha sido considerada como "armonía", la salud también es definida como todo lo contrario, a saber, como "lucha", como "momento de un proceso dialéctico", etcétera (Schmidt, 2010). En efecto, las dos grandes teorías de la salud tanto en la teoría como en filosofía de la medicina contemporáneas contemplan e integran esta pugna de un modo $\mathrm{u}$ otro. Pues, pese a la gran diversidad de teorías de la salud presentes en la literatura, la mayoría de ellas defienden o bien una posición normativista o bien una posición naturalista. Mientras que la postura normativista sostiene que para evaluar el estado de salud y/o enfermedad de una persona se precisa una consideración holística del sujeto en cuestión; la postura naturalista afirma que tal evaluación necesita enfocarse principalmente en elementos anatómicobiológicos. La visión más famosa del enfoque naturalista es defendida por Christopher Boorse.

Boorse, en su Teoría Bioestadística de la Salud (Bio-Statistical Theory), define la enfermedad como una disfunción del funcionamiento normal de un organismo y/o ser vivo. Según él, los conceptos de salud y enfermedad corresponden a conceptos neutros, empíricos y objetivos, en cuanto éstos se basan en funciones o disfunciones biológicas naturales. Por ello, la posición de Boorse considera que la noción de enfermedad es un concepto descriptivo al que escapan todo tipo de valoraciones subjetivas: "The fundamental idea is that a pathological condition is a state of statistically species subnormal biological part-function, relative to sex and age" (2014: 684). La visión naturalista de Boorse define la enfermedad en oposición a la salud, considerando a esta última como el fun- 
cionamiento biológico y específico considerado típico o "normal" del hombre en tanto miembro de la especie homo sapiens. Mientras que la salud de un organismo está dada por el orden natural de un organismo siendo esto aquello que corresponde a su estado biológico normal: "Very important is that the biostatistical theory (BST) is, and always has been, a dynamic count of normal physiology" (2014: 685). Un organismo que vive en virtud de su eficiencia normal típica, debe ser capaz, siguiendo a Boorse, de alcanzar satisfactoriamente dos metas fundamentales: supervivencia y reproducción. La noción de normalidad estadística se ubica por tanto en el centro de su teoría de salud, ya que sólo tomando en cuenta esto es posible determinar si un individuo está sano o enfermo. Según Boorse, un organismo puede estar enfermo en sentido teórico objetivo (a lo que Boorse llama disease) como también en sentido subjetivo (para ello utiliza la palabra illness).

Un ser vivo es normal y está sano si, por consiguiente, sigue la estructura funcional biológica "normal" y típica de su especie. Considerando lo anterior, se evidencia que Boorse comprende el concepto de salud de forma negativa, pues se trata de un concepto que es definido desde la ausencia de la enfermedad, en tanto la enfermedad es constatada como un obstáculo o impedimento del funcionamiento típico y normal del organismo. En este sentido, Boorse define a la salud de la siguiente forma: "Health in a member of the reference class is normal functional ability: the readiness of each internal part to perform all its normal functions on typical occasions with at least typical efficiency" (2014: 684).

La segunda teoría de la salud corresponde a la posición de los normativistas. A este enfoque pertenecen la mayoría de los teóricos de la salud, los cuales tienen en común considerar la salud y la enfermedad como conceptos normativos. Contrario a la posición de Boorse los normativistas argumentan que el diagnóstico médico no consiste en la descripción de datos empíricos, sino que 
dichas descripciones siempre contienen una serie de valoraciones subjetivas, culturales y/o éticas. De acuerdo con los normativistas, para determinar el estado de salud de una persona no es suficiente dar con datos cuantitativos o estadísticos, sino que esto requiere de un diagnóstico holístico, una "evaluation of the general state of the person” (Nordenfelt, 2007: 6). Uno de los enfoques discutidos más seriamente y que pertenece al flanco de los normativistas, corresponde a la Teoria Holistica de la Salud (Holistic Theory of Health, HTH) de Lennart Nordenfelt (Schramme, 2007).

De acuerdo con la teoría de Nordenfelt, la salud no se identifica con la ausencia de la enfermedad, sino que ella refiere a la "capacidad" (ability) de alcanzar "metas vitales" (vital goals). Capacidad y discapacidad constituyen así conceptos fundamentales para determinar el estado de salud de una persona: "Disability is a negative notion presupposing the semantic content of its positive contrary, ability. This gives the analysis of ability a primary place in my theory of health" (Nordenfelt, 2001: 67). En su teoría de la salud, Nordenfelt distingue entre varios conceptos: enfermedad, sufrimiento, dolor e impedimento (o discapacidad) y aboga por la centralidad del concepto de impedimento.

De acuerdo con su planteamiento, una persona debe ser considerada saludable si ella cuenta con determinadas capacidades que le permiten alcanzar una meta vital determinada (y no arbitraria). En este sentido, Nordenfelt define la habilidad como una característica que debe ser evaluada en relación a tres aspectos: al agente en cuestión, a su meta (o metas) respectiva y a las circunstancias en las que aquel agente actúa. Nordenfelt ciertamente no establece una lista concreta de aquello que constituyen "metas vitales", sino que ofrece tres requisitos formales para así determinar subjetivamente su contenido. Nordenfelt define las metas vitales en relación a un bienestar o felicidad a largo plazo: "A is completely healthy if, and only if, A has the ability, given standard circumstances, 
to reach all his or her vital goals" (Nordenfelt, 2007: 7). De este modo, la salud es interpretada como un requisito fundamental para tener una vida satisfactoria, es decir, para alcanzar una buena vida. En relación a lo anterior, una enfermedad no implica un defecto anatómico del cuerpo "normal" como en el caso de la teoría de Boorse, sino que lo realmente determinante debe ser la presencia de una situación percibida como problemática: "My basic idea is that the notion of illness when it comes to humans has its basis in the existence of a perceived problema" (Nordenfelt, 2007: 7). El reconocimiento subjetivo de una situación o estado como problemático constituye pues, el requisito fundamental, siguiendo a Nordenfelt, para buscar ayuda médica.

Luego, y dado que ahora es posible entender qué significan salud y enfermedad en sentido lato, se puede entender mejor la riqueza y pertinencia de la meditación Nietzscheana para describir la relación (agonal) entre hombres y animales a partir de la agonalidad de la relación entre los conceptos de salud y enfermedad. Pues ambas teorías de la salud, a mi juicio, en cuanto dan cuenta de formas distintas de entender y vivenciar la salud, se realizan en modos igualmente diversos. El caso de la enfermedad como fenómeno empírico-positivo y entendido bajo la fórmula naturalista, corresponde, como descrito, a la teoría de Boorse. Esta teoría reafirma una idea dominante en la medicina al menos desde su cientifización a mediados del siglo XIX, a saber, que la medicina responde a un modelo "científico" y "objetivo" responsable de "aplicar" sus conocimientos en el ámbito de la salud humana. Pese a que esta concepción aún tiene un amplio apoyo, incluso en los momentos más ortodoxos en los que la medicina intentó definirse como ciencia fisiológica, es decir, como una disciplina empírica y fundada en la observación del organismo como un conjunto de procesos químico-físicos, ya se hacían sentir críticas ante este modelo por considerarle reduccionista. Sin embargo, y pese a 
todas las críticas que hasta ahora se han planteado a tal forma de entender tanto a la medicina como a la salud y a la enfermedad (gracias a conceptos como medicina individualizada por ejemplo, y que intenta resaltar la individualidad o al menos, especificidad en estratos genéticos para tratar ciertas enfermedades), ella tiene una gran ventaja, pues, con toda las diferencias específicas que podamos tener los humanos, ciertamente permite entender que, en tanto organismos vivientes, enfermamos.

En este sentido, la teoría de Boorse permite establecer una importante cercanía entre animales y humanos. Ambos, independientemente de todas las diferencias que puedan establecerse entre ellos, comparten una vulnerabilidad producto justamente de su común ser biológico. No es trivial, pues, así como hay médicos dedicados a tratar personas, también existen veterinarios para tratar y curar animales. La enfermedad en sentido de un mal interpretado desde aspectos empíricos, es pues un ámbito que acerca lo humano y lo animal.

Sin embargo, considerando la teoría normativista de la salud propia de Nordenfelt nos hayamos frente a una situación completamente distinta: si la salud tiene que ver con la realización de metas vitales y dichas metas deben ser evaluadas en relación al individuo en su totalidad, aquella pareciera enfocarse a otro tipo de comprensión de la salud que acentúa la diferencia entre animales y humanos. En este sentido, salud y enfermedad tienen que ver no tanto con la funcionalidad o disfuncionalidad biológico-anatómica de partes del organismo como en el caso de la teoría naturalista, sino más bien con la realización y/o cumplimiento de metas vitales. Lo saludable, bajo esta perspectiva, sería convergente con una posición nietzscheana, dado que lo saludable no remite a un individuo que posee una constitución biológica promedio con respecto a la propia especie, sino a quien independiente de una posible condición orgánica desfavorable, se auto-interpreta como saluda- 
ble. En este sentido, la visión de salud y enfermedad de Nietzsche se acerca fundamentalmente a la corriente normativista. Para Nietzsche, el hombre es "el animal enfermo" contemplado desde la perspectiva del animal y de su ámbito natural; sin embargo, gracias a esta carencia natural, paradójicamente, puede alcanzar una verdadera sanidad, una sanidad en cierto modo fuera de lo natural; una salud en el mundo humano. El hombre constituye para Nietzsche "el animal enfermo" que, pese a lo paradojal del asunto, justamente debido a lo deficitario de sus instintos, a causa de su condición "enferma” desde el punto de vista biológico, puede construir una sanidad más allá de los límites que impone el terreno de lo natural. Así, la esencia "deficitaria", dicho con Gehlen, de lo humano, se transforma en fuente de una nueva "salud" posible, dotada de sentido y que reacciona como respuesta a su condición "enferma". Lo anterior se relaciona íntimamente con el concepto Nietzscheano de salud y enfermedad expuesto en diversas partes de su obra y que se caracteriza principalmente por ser pluralista, radicalmente individual, dinámico y transfigurador, es decir, que considera incluso la enfermedad como "estimulante del vivir" - "ein Stimulans des Lebens"- Nietzsche nos dirá esto tanto en El caso Wagner (Der Fall Wagner) (1999b: 22) como en algunos fragmentos póstumos (Aurenque, 2016).

\section{Dos conclusiones}

Nietzsche, sin duda alguna, es uno de los filósofos que más valora lo agonal, la lucha en sentido griego, como elemento de crecimiento individual y social. La relación entre salud y enfermedad representa justamente una relación de lucha constante, entre dos estados opuestos que parecen impensables en propiedad sin la mutua referencia. Dado lo anterior, se explica que tome esta diada para explicitar, por un lado, su tesis antropológica-crítica y, por 
otro, para fortalecer una vez más su filosofía monista donde los contrarios siempre bajo alguna perspectiva pueden tener cabida. La definición del hombre como "el animal enfermo" da cuenta de la doble perspectiva con la cual debemos tratar de entender la naturaleza humana: cuando la salud es considerada comparativamente entre especies y de forma naturalista (siguiendo a Boorse), entendemos el contexto desde el que Nietzsche denomina al hombre como "el animal enfermo". Sin embargo, esa "enfermedad" en realidad no refiere a un estado biológico-naturalista concreto de un individuo, sino a la dificultad existencial de vivir desorientado en medio de la naturaleza. En efecto, si miramos con atención veremos que ninguna especie animal tiene dificultades tan enormes como el ser humano respecto de la reproducción y de la sobrevivencia; mejorando estas últimas sola y exclusivamente en virtud de la creación de técnicas, ciencias y un mundo cultural capaz de hacer frente a la condición deficitaria humana. En este sentido, la salud humana poco tendría que ver con la salud de los animales postuladas por Boorse. Ahí, en plena carencia el ser humano se auto-transforma en animal "sano"; dicha sanidad si bien no reniega de lo natural (pues la vulnerabilidad natural es un límite inalienable) lo reubica, re-articula, re-significa en otro ámbito, en un mundo construido y distinto a lo dado naturalmente, en el cual la falencia orgánica no es preponderante, sino incluso vista como fortaleza cognitiva. En esto último, en la posibilidad de reasignar valores de salud a lo biológicamente enfermo, se acerca la antropología de Nietzsche especialmente a la noción normativista de Nordenfelt. Es más, la visión de la salud de Nietzsche es muy similar a visiones contemporáneas y críticas del "modelo médico", que precisamente abogan por considerar a las discapacidades no como males o enfermedades, sino como formas de expresión de identidad cultural. 
A modo de conclusión, quisiera destacar brevemente al menos dos aspectos que me parecen valiosos del análisis de la antropología negativa de Nietzsche en relación a la pregunta por la relación entre humanos y animales:

1) La primera consecuencia, sin duda fundamental, que se desprende de la agonalidad entre lo humano y lo animal a partir de criterios de salud y enfermedad, tiene que ver con la extraordinaria puesta al descubierto que hace Nietzsche del carácter, como quisiera llamar aquí, ontológico-moralizador de una metafísica racionalista. Pues, pese a que Nietzsche en su antropología negativa critica a lo animal por considerarle deficitario y desligado de sus instintos naturales, esto permite desde el plano genealógico justificar su "hacerse hombre" desde la enfermedad. A diferencia de la tradición judeo-cristiana, así como de corrientes platónico-aristotélicas e incluso de líneas romántico-filosóficas, el hombre según Nietzsche no es definido por un ideal metafísico, sino descrito desde lo que ha sido fácticamente, desde el reconocimiento de su fisiología. O dicho en otros términos: mientras que la tradición metafísica se caracteriza por defender nociones antropológicas basadas en la confusión entre hombre como factum y el hombre como postulat, Nietzsche lo describe desde una factidad-amoral. Esto implica que mientras las categorías antropológicas de corte metafísico-racionalistas definen a lo humano desde lo que debería ser (como ser racional en las diversas formas de entender esto: como ser político y racional al modo aristotélico o como ser de la ley moral kantiana por ejemplo), y así, desde un plano que lo desliga de su fisiología por considerarle un ámbito menos esencial que sus capacidades cognoscitivas (p. ej. Kant niega el rol de la fisiología para la antropología filosófica de forma tajante), Nietzsche muy por el contrario integra en su filosofía los avances científicos de su tiempo y construye una antropología crítica basada en eso. Por tanto, él incluirá no sólo conocimientos de la fisiología humana imperantes 
en el siglo XIX en su reflexión sobre lo humano y lo animal -con el desarrollo de la anatomía, de la patología morfológica y luego de la patología celular de Rudolf Virchow, etc.- y que se caracterizan por considerar el organismo humano enmarcado en la teoría evolutiva de Charles Darwin. Nietzsche es representante de un pensar de lo humano distinto, que no le define como ideal de sí mismo, que lo valora acentuando su animalidad, pero que también incluso lo denigra a algo que debe ser superado.

2) La segunda consecuencia que me interesa destacar consiste en que gracias a la definición antropológica negativa de lo humano como "el animal enfermo", Nietzsche esboza una dualidad en la posible forma de ser "enferma" del hombre que, por un lado, permite pensar y hasta cierto punto integrar las dos teorías actuales de la salud más importantes en la teoría de la medicina y, por otro, invita a reflexionar las cercanías y distancias de lo humano con lo animal de una forma novedosa, descriptiva e integradora. Ya que, al tomar los conceptos de salud y enfermedad como ámbitos de reflexión, tal como fue expuesto en el presente trabajo, encontramos una forma ajena a la tradición moralizadora metafísica de acceder y comprender la relación agonal entre lo humano y lo animal, sin por ello eludir u obviar sus diferencias. En este sentido, el proyecto negativo-antropológico de Nietzsche ofrece, a fin de cuentas, una metafísica distinta a la metafísica tradicional que aboga por pensar la relación humano-animal no desde la oposición frontal y estática (como una grieta que los separe inexorablemente); por el contrario, y dada la conexión innegable de éstos a partir de su común raíz biológica-natural, ambos no son considerados desde una mera oposición, sino que su relación es más bien dinámica, contextual e incluso transformadora. 


\section{Referencias}

Aurenque, Diana /Friedrich Orsolya (eds.), 2013, Medizinphilosophie oder philosophische Medizin? Ethische Beiträge zu Herausforderungen technisierter Medizin, Frommann/Holzboog Verlag, Stuttgart/Bad Cannstatt.

Aurenque, Diana, 2013a, "Medizinische Philosophie: Schopenhauers und Nietzsches Gesundheitslehren", Ärzteblatt $B a$ den-Württemberg ( $\ddot{B} B W)$, núm. 02, pp. 56-58.

, 2013b, "Über Nietzsches medizinische Deutung der Moral: Das Leiden als Ursprung der Moralität bei Schopenhauer und Nietzsche", en Birnbacher, Dieter/Sommer, Andreas Urs (ed.), Moralkritik bei Schopenhauer und Nietzsche, Reihe, Beiträge zur Philosophie Schopenhauers, Königshausen \& Neumann, Würzburg, pp. 45-60.

, 2016, "Nietzsche und die unzählige[n] Gesundheiten des Leibes", en O. Friedrich, D. Aurenque, G. Assadi y S. Schleidgen, Nietzsche, Foucault und die Medizin, Transcript-Verlag, Bielefeld, pp. 23-37.

Boorse, Christopher, 1977, "Health as a Theoretical Concept", Philosophy of Science, núm. 44, pp. 542-573. , 2014, "A Second Rebuttal On Health", Journal of Medicine and Philosophy, núm. 39, pp. 683-724.

Brusotti, Marco, 2001, "Wille zur Macht, Ressentiment, Hypnose. ,Aktiv' und ,reaktiv' in Nietzsches Genealogie der Moral", Nietzsche-Studien, núm. 30, pp. 107-132.

Cherlonneix, Laurent, 2002, Philosophiemédicale de Nietzsche: la connaissance, la nature, Harmattan, París.

Friedrich, Orsolya, Diana Aurenque, Galia Assadi y Sebastian Schleidgen (eds.), 2016, Nietzsche, Foucault und die Medizin, Transcript/Verlag, Bielefeld. 
Gehlen, Arnold, 2016, Der Mensch: Seine Natur und seine Stellung in der Welt, Vittorio Klostermann, Frankfurt.

Jaspers, Karl, 1950, Nietzsche. Einführung in das Verständnis seines Philosophierens, núm 3, de Gruyter, Berlin.

Lemm, Vanessa, 2012, Nietzsches Philosophie des Tieres, Diaphanes Verlag, Berlin/Zurich. Chile.

2014, Nietzsche y el devenir de la vida, FCE, Santiago de , 2016, "Biopolitische Betrachtungen zur Figur des Arztes in Nietzsches Philosophie", en Nietzsche, Foucault und die Medizin, Transcript Verlag, Bielefeld, pp. $183-201$.

Long, Thomas, 1990, "Nietzsche's Philosophy of Medicine", Nietzsche-Studien, núm. 19, pp. 112-128.

Nietzsche, Friedrich, 1999a, Der Fall Wagner, Kritische Studienausgabe (KSA) 6, G. Colli y M. Montinari (eds.), DTV/de Gruyter, Berlín.

, 1999b, Der Antichrist. KSA 6, G. Colli y M. Montinari (eds.), DTV, Berlín.

, 1999c, Also sprach Zarathustra. KSA 4, G. Colli y M. Montinari (eds.), DTV, Berlín.

, 1999d, Zur Genealogie der Moral. KSA 5, G. Colli y M. Montinari (eds.), DTV, Berlín.

, 2014, La Genealogía de la Moral, Andrés Sánchez Pascual (trad.), Alianza, Madrid.

Nordenfelt, Lennart, 2001, Health, Science, and Ordinary Language, Rodopi, Amsterdam.

, 2007, "The Concepts of Health and Illness Revisited", Medicine, Health Care and Philosophy, núm.10, p. 5. 
Real Academia Española, versión electrónica, significado palabra "agonal". Disponible en: www.rae.es (Consultado: 25/ IV/2016).

Ruffing, Reiner, 2008, Bleibt der Erde treu! Nietzsches Hymnus auf das Leben, Franz Steiner, Stuttgart.

Scheler, Max, 1997, El puesto del hombre en el Cosmos, José Gaos (trad.), Ed. Losada, Buenos Aires.

Schipperges, Heinrich, 1975, Am Leitfaden des Leibes. Zur Anthropologik und Therapeutik Friedrich Nietzsches, Klett, Stuttgart.

, 1981, Kosmos Anthropos. Entwürfe zu einer Philosophie des Leibes, Klett/Cotta, Stuttgart.

, 1985, Homo patiens. Zur Geschichte des kranken Menschen, Piper, München/Zürich.

Schmidt, Josef M., 2010, "Gesundheit! - Geschichte und Konzepte des Leitbegriffs der Medizin”, Wiener klinischeWochenschrift, núm. 122, pp. 538-542.

Schramme, Thomas, 2007, "A Qualified Defence of a Naturalist Theory of Health", Medicine, Health Care and Philosophy, núm. 10, pp. 11-17.

, 2009, "Nietzsche, der Übermensch und Transhumanismus", en Der neue Mensch? Enhancement und Genetik, Alber, Freiburg/München, pp. 127-144.

, 2011, "Nietzsche", en Geschichte der Bioethik. Eine Einführung, Mentis, Paderborn, pp. 213-229. 